\title{
II.
}

Aus dem I anatomisehen Institut (Hofrat Prof. Dr. E. Zucker$\mathrm{k}$ andl) der k. k. Universität Wien.

\section{Beitrag zur Kenntnis der Knochenneubildung im Mittelohre bei chronischen Eiterungen.}

Von

Dr. Hugo Frey,

em. Assistenten der $k, k$. Universitäts-Ohrentlinil (Hofrat Prol. Dr. A. Politzer) in Wien.

(Nit 1 Abbildung.)

Die Neubildung von Knochensubstanz tritt am entwickelten Schläfenbein, wenn wir von der typischen Otosklerose absehen, in der Regel in zwei Formen auf, und zwar diffus als 0 steosklerose, vorwiegend im Inneren des Knoehens, und eireumseript in Gestalt von Osteomen, vorzugsweise an der Oberfläche des Knochens.

Wiewohl das sehr differente Verhalten der Knochensubstanz des Sebläfenbeins in Bezug anf seine Struktur sehon lange bekannt war, hat doch erst die operative Aera der Otiatrie das volle Verständnis und Interesse für diese Beziehungen gezeitigt.

Die Entstehung der Oste osklerose beruht, wie mit Sicherheit angenommen werden kann, auf dem Übergreifen der Entzündungs- und Eiterungsprozesse der Mittelohrsehleimhant auf den Knochen. Es kommt dabei vielfach zu einer Eburnisation desselben, wobei die Bildung der neven Knoehensubstanz sich nieht nur auf das Innere des Knochens besehränken muß, sondern wobei auch an der Oberfläche durch Apposition eine oft wesentliche Zunahme des Umfanges erreicht wird 1).

Dort, wo die Oberfläche des Knochens die Begrenzung von Hohlräumen bildet, wie z. B. im äußeren Gehörgang und im An-

1) Schwartze, "Pathologische Anatomie des Ohres", in Klebs: Handbuch der pathologischen Anatomie. 1878. 
trum mastoideum, bewirkt die Massenzunahme der Knocheusubstanz eine mitunter beträehtliche Verkleinerung und Verengerung dieser Räume, die zu vollständiger Obliterierung führen kann.

Man hat es hier mit einem Ausgang der Knochenentzündung zu tun, der im allgemeinen als ein partieller Heilungsvorgang im pathologisch-anatomischen Sinne zu betrachten sein wird. Praktisch genommen ist es aber sehr fraglich, ob dieser Prozess in irgend einer. Weise imstande ist, das Fortschreiten der Eiterung im Knochen mit Erfolg zu verhindern, oder die Gefahr der Fortleitung der Eiterung auf das Schädelinnere aufznhalten.

Die Mehrheit der Autoren ist sogar der Meinung, dab die Osteosklerose eine ungünstige Komplikation der chronisehen Otitiden vorstelle. Jedenfalls ist sie eine der häufigsten und die Angaben der Autoren differieren nur in Bezug auf den Grad dieser Häufigkeit.

So fand Grunert ${ }^{1}$ ) Osteosklerose in 10 Proz. aller operierten Fälle mit einfacher Eiterung und in 13,5 Proz. der mit Cholesteatom komplicierten.

Körner ${ }^{2}$ beobachtete sie sogar in 36 Proz. der einfachen und in 69 Proz. der Cholesteatomfälle. Er erklärt die Differenz zwischen seinen und Grunerts Zahlen damit, daß er auch geringere Grade der Knochenverdichtung als Grunert noch mit in Rechnung zog.

Bezold ${ }^{3}$ ) meint überdies, daß sie eine geradezu regelmäBige Begleiterscheinung der chronischen Eiterungen bilde.

Diese Mitteilungen beziehen sich jedoch nur anf die Veränderungen im Warzenfortsatz.

Das Vorkommen entsprechender hyperostotischer Prozesse im Gehörgang wird schon seltener, wenn auch noch immer recht häufig erwähnt, noch viel seltener eine hochgradige Verkleinerung des Antrum.

Vollkommene Obliterierung des Antrum sah Grune ${ }^{t^{4}}$ ) nur in 2 Fällen.

1) Grunert, Beitrag zur operativen Freilegung der Mittelohrräume. Dieses Archiv. Bd. XL.

2) Körner, Die eitrigen Erkrankungen des Schläfenbeins. Wiesbaden 1899 . S. $71 \mathrm{ff}$.

3) Bezold, Überschau uber den gegenwärtigen Stand der Ohrenheilkunde. 1895 .

4) Grunert, 1. c. 
Auch Sebwartze $\left.{ }^{1}\right)$ und Bezold 2) erklären sie als seltenen Befund.

Politzer ${ }^{3}$ ) weist anf sie als ein sehr vereinzeltes Vorkommnis hin.

Was nun die zweite Form der Knochenneubildung, die umschriebenen 0 steome, betrifft, so wird ihr Vorkommen, mit Ausnahme derjenigen der Gehörgangswand, nicht oft beobachtet.

C. 0 . Weber ${ }^{4}$ ) zählt im ganzen 3 Osteome der Oberfläehe und 8 des äußeren Gehörganges auf. Von den zweitgenannten ist es zum Teil wahrseheinlieh, daß sie entzündlichen Prozessen ihre Entstehung verdanken.

Während nun die Exostosen- und Hyperostosenbildung im äußeren Gehörgang seither als ein verhältnismäßig häufiger Befund (Hessler $\left.\mathrm{r}^{5}\right)$ ) sich herausgestellt hat und vielfach Hand in Hand mit der Osteosklerose geht, hat die Kasuistik der Osteome an anderer Stelle des Schläfenbeins nur eine geringe Vermebrung erfahren (Politzer ${ }^{6}$ ), Gruber ${ }^{7}$ )). lnsoweit diese Osteome die Außenfläche des Warzenfortsatzes und der Schuppe betreffen, dürften sie mit den auf entzündlicher Basis entstandenen Knochenhypertrophien nichts zu tun haben.

Ein gleichzeitiges Vorkommen von Osteosklerose im Inneren des Knochens und exostotischem Prozesse an der Oberfläche wurde von E. Zuckerkand ${ }^{8}$ ) beschrieben. Er fand bei einem Individuum am rechten Schläfenbein auf der äuberen Fläche des Warzenfortsatzes in der Incisura mastoidea, in der Fissura sigmoidea, um die Austrittsöffnung des Fallopisehen Ganges und auf der Gehörgangswand zahlreiche weiße, retikuläre Osteophytenlager, und dasselbe im Inneren des Warzenfortsatzes, an beiden Schläfenbeinen eines anderen Individuums einen ähnlichen Befund, jedoch verbunden mit Resten cariöser Zerstörung. Die

1) Schwartze, Casuistik zur chirurgischen Eroffnung des Warzenfortsatzes. Dieses Archiv. Bd. XII. $157 \%$.

2) Bezold, "Die Erkrankungen des Warzenteiles", in Schwartzes Handbuch der Ohrenheilkunde. II. S. 328.

3) Politzer, Lehrbuch der Ohrenheilkunde. 4. Aufl. 1901. S. 456.

4) C. O. Weber, Die Knöchengeschwülste in anatomischer und praktischer Beziehung. Bonn 1856. 1. Abt. S. 37 ff.

5) Hessler, dieses Archiv. 1896. Bd. XLI. S. 176.

6) Politzer, l. c. S. 519.

i) Gruber, Lehrbuch der Ohrenheilkunde. 2. Aufl. S. 375.

8) Emil Zuckerkandl, Über eine seltene Ausbreitungsweise von Osteophyten im Schläfenbein. Monatsschr. f. Ohrenh. 1880. S. 34 ff. 
Paukenhöhle war hingegen in allen 3 Fällen vollständig intakt.

Diese Verschonung der Paukenhöhle durch den WucherungsprozeB des Knochens scheint überhaupt die Regel zu sein. Ioh finde in der Literatur nur ganz wenige Fälle verzeichnet, in denen die Paukenhöhle selbst zum Sehauplatz des Knoehenwachstums wurde.

So berichtet Tröltsch ${ }^{1}$ ) über einen Fall, in dem man das Promontorium etwas verdickt, das runde Fenster und den zu ihm führenden Kanal zwar von normaler Größe, jedoch eine spitze Exostose an dem die Nische des runden Fensters deckenden Vorsprung fand.

Moos und Steinbrtig g $\mathrm{e}^{2}$ ) sahen in einem Falle das ganze Felsenbein hyperostotisoh, an der hinteren Pyramidenfläche und an ihrer Basis buckelige Auftreibungen, außerdem an der hinteren Felsenbeinfläche zwei umschriebene, stark vorspringende Exostosen und eine eben solche an der hinteren Pyramidenkante. Der Gehörgang war stark verengt, der Boden der Trommelhöhle verdickt, das Ostium tympanicum der Tube außerordentlich verengt und das runde Fenster knöchern verschlossen.

Des weiteren fand Grunert ${ }^{3}$ ) in zwei Fällen das Lumen der Paukenhöble durch Hyperostose der Mittelohrwandungen fast ganz aufgeboben, und Habermann ${ }^{4}$ ) in einem Falle eine hochgradige Hyperostose des Knochens an seiner Paukenböhlenseite, vom ovalen Fenster herab bis zur Membran des runden sich erstreekend, wodurch die Nische des letzteren in ihrer vorderen Hälfte fast vollständig verschlossen wurde. In zwei anderen Fällen sah er die Paukenhöhlenwand durch Hyperostosenbildung buckelig und höckerig geworden.

Das Vorkommen von Exostosenbildung an der medialen Seite des Amboh, allerdings aus unbekannter Ursache, habe ich einmal beobachtet ${ }^{5}$ ).

Im Folgenden möchte ich nun dje Aufmerksamkeit auf ein

1) Tröltsch, Anatomische Beiträge zur Ohrenheilkunde. Virchows Arehiv. Bd. XVII. S. 28.

2) Moos und Steinbrügge, Hyperostosen- und Exostosenbildung usw: Zeitschr. f. Ohrenheilk. Bd. XI. S. $48 \mathrm{ff}$,

3) Grunert, 1. c. S. 195 u. 201.

4) Habermann, Zur Pathologie der chronischen Mittelohrentzündung und des Cholesteatoms des äußeren Gehörgangs. Dies. Arch. Bd. L. S. 236 ff.

5) Frey, Bericht über eine seltene Abnormität am menscblichen Amboß. Dieses Archiv. Bd. LVIII. 1903. 
Vorkommnis lenken, das bisher noch nicht beschrieben wurde und daher wohl ein recht seltenes zu sein scheint. Es handelt sich um den Befund ausgedehnterer umschriebener Exostosenbildung an der lateralen Seite des horizontalen Bogenganges. Ich wa in der Lage, den genannten Befund dreimal zu erheben, und zwar zweimal am Lebenden und einmal am macerierten Präparat.

Die ersten 2 Falle betrafen Patienten der hiesigen Universitätsohrenklinik. Bei beiden wurde wegen chroniseher Eiterung and Cholesteatombildung von Herrn Hofrat Politzer die totale Freilegung der Mittelohrräume vorgenommen. Beide Male füllten große Cholesteatomknollen die Trommelhöhle, den Kuppelraum und das Antrum fast vollständig, zum Teil auch den Warzenfortsatz aus. Nach ihrer Entfernung zeigte sich auf dem horizontalen Bogengange, und zwar an der am meisten lateral vorspringenden Stelle ein umschriebener, etwas überhanfkorngroßer, breit aufsitzender und harter knöcherner Vorsprung. In dem einen dieser beiden Fälle trug dieser Vorsprung noch zwei kleinere Prominenzen.

Das Präparat des dritten Falles gelangte durch die Güte des Herrn Prof. Dr. J. Tandler, Prosector der I. anatomischen Lehrkanzel an der Universität Wien, in meine Hand. Da sich das Objekt zufällig unter dem macerierten Materiale des anatomisehen Institutes fand, ist von der Krankengeschichte und dem Befunde am Lebenden niohts bekannt.

Die nachfolgende Beschreibung wird durch die beigegebene Abbildung verdeutlicht.

An dem im übrigen normal gebanten Sehädel zeigt sich der Gehörgang und die Trommelböhle der rechten Seite ganz normal. Linkerseits sieht man Folgendes:

Die obere und hintere Gehörgangswand fehlen vollständig. Die Gehörgangsöffnung ist dadurch zu dem unregelmäßig begrenzten Eingang einer Höhle erweitert, dessen vertikaler Durchmesser $22 \mathrm{~mm}$, sein horizontaler $14 \mathrm{~mm}$ beträgt. Nach innen vom Eingang vergrößert sich der Hohlraum noch so weit, daß der Knochen nach vorn', oben und hinten allseits bis an die Tabula vitrea freigelegt ist. $\mathrm{Er}$ ist hier therall glatt, wie abgeschliffen.

An der der hinteren Sehädelgrube zugehörigen, hinteren Wand der Höhle (der früheren hinteren Antrumwand) findet sich eine größere und eine kleine Durehbruchsstelle gegen die Schädelhöhle (hS). An der inneren Trommelhöhlenwand sieht man eine 


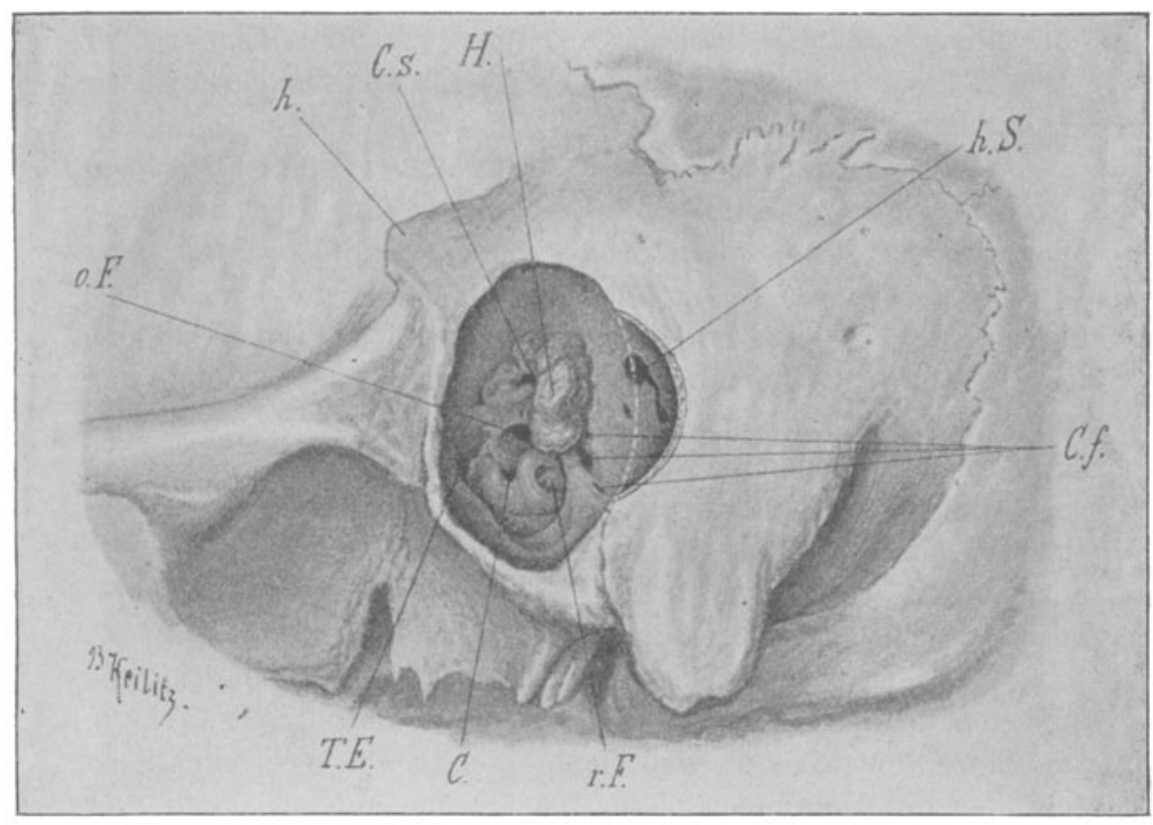

$o . F$. ovales Fenster, $h$. Exostose am Jochfortsatz, $C^{x} s$. eröffneter horizontaler Bogengang, $H$. Exostose, dem Rogengang aufsitzend, $h . S$. Durchbruch gegen die hintere Schädelgrube, $C . f$. Offnungen gegen den Facialkanal, $r . F$. rundes Fenster, C. Offnung gegen die Schnecke, T.E. tympanales Tubenostium.

(Die weiß punktierte Linie zeigt die wirkliche Begrenzung der Öffnung, das nach rechts von ibr liegende Stttck ist in der Zeichnung als entfernt angenommen, um die inneren Partien zur Ansicht zu bringen.)

Öffnung von $1^{1 / 2} \mathrm{~mm}$ Durchmesser im Centrum des Promontorium, die direkt in die Schnecke führt (C). Die Nisehe des runden Fensters $(r F)$ ist dentlich erweitert, das ovale Fenster $(o F)$ sieht an seinem unteren Rande wie ausgefressen aus, von ihm verläuft nach abwärts über das Promontorium eine Furehe bis zu dem früher erwähnten Loch im Promontorium. Dureh dieses wie auch durch die Fenster selbst kann man deutlich cariös veränderte Knochenpartien sehen.

Das Lumen des horizontalen Bogenganges $(\mathrm{Cs})$ ist auf etwa $2 \mathrm{~mm}$ Länge* eröffnet, drei kleinere öffnungen (C F) führen zum Canalis facialis. Rund um den borizontalen Bogengang findet sich nun eine deutlich vorspringende wallartige Knochenwucherung. Dieselbe ist vorn oben sowie unten in ihrem vorderen Anteil von bloß $2 \mathrm{~mm}$ Höhe, nach hinten nimmt sie an Höhe bedeutend zu und formt sich zu einer weit in den Mittelohrraum 
vorspringenden, mehrfach gewulsteten, höckerigen Exostose, die über den Facialkanal nach abwärts iuberhängt. Der Eingang der Tube (TE) ist erweitert. An seinem oberen Ende ist ein kariöser Defekt der Wand sichbar.

Weiterhin findet sich außen, an der oberen Wurzel des Jochfortsatzes eine dünne, blattförmige Exostose (h). Der aufgesägte Warzenfortsatz erwies sich als hochgradig sklerotisch.

Die beschriebenen Veränderungen können nicht anders als durch eine chronische Mittelohreiterung mit Übergreifen auf den Knochen, kariöser Zerstörung desselben und Cholesteatombildung erklärt werden, das letztere deshalb, weil nur durch ein solches so enorme, glattwandige Höhlen geschaffen werden.

Nun zu den Exostosen. Abgesehen von dem Interesse, das ihr bis jetzt noeh nicht beschriebenes Vorkommen an dieser Stelle beansprucht, verdienen sie auch eine gewisse Aufmerksamkeit aus den gleich za erörternden Gründen.

Wir sehen sie hier neben dem Zerstörungsprozeß im Knochen auftreten, ja aus dem zerstörten Knochen selbst hervorwachsen. Der beste Beweis dafür, daß sie hier in keiner Weise das Fortschreiten der Eiterung und ihr Übergreifen auf das innere Ohr und die Schädelhöhle hinderten. Im Gegenteile. Wir müssen annehmen, dah sie erst dann entstanden sind, als der Knochen in seiner Substanz selbst erkrankt war, daß also hier der Appositionsproze $B$ dem Zerstörungsproze $B$ nicht vorangegangen, sondern gefolgt ist. Finden wir ihn ja am ausgeprägtesten gerade an der Stelle der größten Zerstörung, woraus hervorgeht, daß die Neubildung des Knochens auch schon ihrer Entstehungszeit nach nicht mehr imstande ist, einen Schutz für das umgebende Gewebe gegen das Eindringen der Eiterung zu bilden.

Ein ähnliches, gleichzeitiges Vorkommen von Einschmelzungs - und Neubildungsprozessen im Knochen habe ich auch im Hammer-Amboßgelenk beobachtet 1 ).

Es ist wahrscheinlich, dab dieses, hier durch das Präparat illustrierte Verhalten auch bei der entzündlichen Knochenneubildung in den anderen Teilen des Schläfenbeins besteht, womit die Ansicht derjenigen Autoren bestätigt wird, welche der Osteosklerose als einer wirksamen Schutzvorrichtung "des Organismus keinen Wert zuerkennen wollen.

Schwieriger fällt die Erklärung des Knochenvorsprunges am

1) Frey, Die Ankylose des Hammer-Amboßgelenkes. Dieses Archiv. Bd. LXI. 1904. 
Jochfortsatz. Es ist immerhin möglich, dab es sich hier um den zufälligen Befund einer einfachen anatomischen Abnormität handle. Andererseits wäre es immerhin auffallend, daß eine so gestaltete Varietät ein ganz vereinzeltes Vorkommnis bilden sollte, und es ist derartiges, soweit ich die Literatur tiberblicken kann, bisher noch nirgends beschrieben oder abgebildet worden, auch konnte ich in der reichhaltigen Schädelsammlung des hiesigen anatomischen Museums eine gleiche oder ähnliche Bildung auch nicht einmal andeutungsweise auffinden. Es liegt daher die Möglichkeit ror, dah auch hier die Ursache der Knochennenbildung in der knapp unter der betreffenden Stelle vorhandenen Erkrankung und Einsohmelzung des Knochens gelegen sei. Auf die Form der Exostose mag vielleicht der Zug des an dieser Stelle inserierenden Temporalmuskels von einigem Einflub gewesen sein.

Zum Schlusse möehte ich mir erlauben, Herrn Hofrat Prof. Zuckerkandl und Herr Prosektor Prof. Tandler für die gütige Überlassung des Objektes meinen besten Dank abzustatten. 